\title{
Metric Dimension in fuzzy(neutrosophic) Graphs-V
}

\author{
Henry Garrett \\ Independent Researcher \\ DrHenryGarrett@gmail.com \\ Twitter's ID: @DrHenryGarrett | @DDrHenryGarrett.wordpress.com
}

\begin{abstract}
In this study, the term dimension is introduced on fuzzy(neutrosophic) graphs. The classes of these specific graphs are chosen to obtain some results based on dimension. The types of crisp notions and fuzzy(neutrosophic) notions are used to make sense about the material of this study and the outline of this study uses some new notions which are crisp and fuzzy(neutrosophic).
\end{abstract}

Keywords: Fuzzy Graphs, Neutrosophic Graphs, Dimension

AMS Subject Classification: 05C17, 05C22, 05E45

\section{Background}

To clarify about the definitions, I use some examples and in this way, exemplifying has key role to make sense about the definitions and to introduce new ways to use on these models in the terms of new notions. The concept of complete is used to classify specific graph in every environment. To differentiate, I use an adjective or prefix in every definition. Two adjectives "fuzzy" and "neutrosophic" are used to distinguish every graph or classes of graph or any notion on them.

$G:(V, E)$ is called a crisp graph where $V$ is a set of objects and $E$ is a subset of $V \times V$ such that this subset is symmetric. A crisp graph $G:(V, E)$ is called a fuzzy graph $G:(\sigma, \mu)$ where $\sigma: V \rightarrow[0,1]$ and $\mu: E \rightarrow[0,1]$ such that $\mu(x y) \leq \sigma(x) \wedge \sigma(y)$ for all $x y \in E$. A crisp graph $G:(V, E)$ is called a neutrosophic graph $G:(\sigma, \mu)$ where $\sigma=\left(\sigma_{1}, \sigma_{2}, \sigma_{3}\right): V \rightarrow[0,1]$ and $\mu=\left(\mu_{1}, \mu_{2} \cdot \mu_{3}\right): E \rightarrow[0,1]$ such that $\mu(x y) \leq \sigma(x) \wedge \sigma(y)$ for all $x y \in E$. A crisp graph $G:(V, E)$ is called a crisp complete where $\forall x \in V, \forall y \in V, x y \in E$. A fuzzy graph $G:(\sigma, \mu)$ is called fuzzy complete where it's complete and $\mu(x y)=\sigma(x) \wedge \sigma(y)$ for all $x y \in E$. A neutrosophic graph $G:(\sigma, \mu)$ is called a neutrosophic complete where it's complete and $\mu(x y)=\sigma(x) \wedge \sigma(y)$ for all $x y \in E$. An $N$ which is a set of vertices, is called fuzzy(neutrosophic) cardinality and it's denoted by $|N|$ such that $|N|=\Sigma_{n \in N} \sigma(n)$. A crisp graph $G:(V, E)$ is called a crisp strong. A fuzzy graph $G:(\sigma, \mu)$ is called fuzzy strong where $\mu(x y)=\sigma(x) \wedge \sigma(y)$ for all $x y \in E$. A neutrosophic graph $G:(\sigma, \mu)$ is called a neutrosophic strong where $\mu(x y)=\sigma(x) \wedge \sigma(y)$ for all $x y \in E$. A distinct sequence of vertices $v_{0}, v_{1}, \cdots, v_{n}$ in a crisp graph $G:(V, E)$ is called crisp path with length $n$ from $v_{0}$ to $v_{n}$ where $v_{i} v_{i+1} \in E, i=0,1, \cdots, n-1$. If one edge is incident to a vertex, the vertex is called leaf. A path $v_{0}, v_{1}, \cdots, v_{n}$ is called fuzzy path where 
$\mu\left(v_{i} v_{i+1}\right)>0, i=0,1, \cdots, n-1$. A path $v_{0}, v_{1}, \cdots, v_{n}$ is called neutrosophic path where $\mu\left(v_{i} v_{i+1}\right)>0, i=0,1, \cdots, n-1$. Let $P: v_{0}, v_{1}, \cdots, v_{n}$ be fuzzy(neutrosophic) path from $v_{0}$ to $v_{n}$ such that it has minimum number of vertices as possible, then $d\left(v_{0}, v_{n}\right)$ is defined as $\Sigma_{i=0}^{n} \mu\left(v_{i-1} v_{i}\right)$. A path $v_{0}, v_{1}, \cdots, v_{n}$ with exception of $v_{0}$ and $v_{n}$ in a crisp graph $G:(V, E)$ is called crisp cycle with length $n$ for $v_{0}$ where $v_{0}=v_{n}$. A cycle $v_{0}, v_{1}, \cdots, v_{0}$ is called fuzzy cycle where there are two edges $x y$ and $u v$ such that $\mu(x y)=\mu(u v)=\bigwedge_{i=0,1, \cdots, n-1} \mu\left(v_{i} v_{i+1}\right)$. A cycle $v_{0}, v_{1}, \cdots, v_{0}$ is called neutrosophic cycle where there are two edges $x y$ and $u v$ such that $\mu(x y)=\mu(u v)=\bigwedge_{i=0,1, \cdots, n-1} \mu\left(v_{i} v_{i+1}\right)$. A fuzzy(neutrosophic) cycle is called odd if the number of its vertices is odd. Similarly, a fuzzy(neutrosophic) cycle is called even if the number of its vertices is even. A set is $\mathbf{n}$-set if its cardinality is $n$. A fuzzy vertex

Table 1. Crisp-fying, Fuzzy-fying and Neutrosophic-fying

\begin{tabular}{l|lcc|l}
\hline & Crisp Graphs & Fuzzy Graphs & Neutrosophic Graphs & \\
\hline Crisp Complete & Fuzzy Complete & Neutrosophic Complete & \\
Crisp Strong & Fuzzy Strong & Neutrosophic Strong & \\
Crisp Path & Fuzzy Path & Neutrosophic Path & \\
Crisp Cycle & Fuzzy Cycle & Neutrosophic Cycle & \\
\hline
\end{tabular}

set is the subset of vertex set of (neutrosophic) fuzzy graph such that the values of these vertices are considered. A fuzzy edge set is the subset of edge set of (neutrosophic) fuzzy graph such that the values of these edges are considered. Let $\mathcal{G}$ be a family of fuzzy graphs or neutrosophic graphs. This family have fuzzy(neutrosophic) common vertex set if all graphs have same vertex set and its values but edges set is subset of fuzzy edge set. A (neutrosophic) fuzzy graph is called fixed-edge fuzzy(neutrosophic) graph if all edges have same values. A (neutrosophic) fuzzy graph is called fixed-vertex fuzzy(neutrosophic) graph if all vertices have same values. A couple of vertices $x$ and $y$ is called crisp twin vertices if either $N(x)=N(y)$ or $N[x]=N[y]$ where $\forall x \in V, N(x)=\{y \mid x y \in E\}, N[x]=N(x) \cup\{x\}$. Two vertices $t$ and $t^{\prime}$ are called fuzzy(neutrosophic) twin vertices if $N(t)=N\left(t^{\prime}\right)$ and $\mu(t s)=\mu\left(t^{\prime} s\right)$, for all $s \in N(t)=N\left(t^{\prime}\right) \cdot \max _{x, y \in V(G)}|E(P(x, y))|$ is called diameter of

Table 2. Crisp-fying, Fuzzy-fying and Neutrosophic-fying

\begin{tabular}{l|lcc|l}
\hline & Crisp Vertex Set & Fuzzy Vertex Set & Neutrosophic Vertex Set & \\
\hline Crisp Edge Set & Fuzzy Edge Set & Neutrosophic Edge Set & \\
Crisp Common & Fuzzy Common & Neutrosophic Common & \\
Crisp Fixed-edge & Fuzzy Fixed-edge & Neutrosophic Fixed-edge & \\
Crisp Fixed-vertex & Fuzzy Fixed-vertex & Neutrosophic Fixed-vertex & \\
Crisp Twin & Fuzzy Twin & Neutrosophic Twin & \\
\hline
\end{tabular}

$G$ and it's denoted by $D(G)$ where $|E(P(x, y))|$ is the number of edges on the path from $x$ to $y$. For any given vertex $x$ if there's exactly one vertex $y$ such that $\min _{P(x, y)}|E(P(x, y))|=D(G)$, then a couple of vertices $x$ and $y$ are called antipodal vertices. For using material look at [1-15].

\section{Definitions}

We use the notion of vertex in fuzzy(neutrosophic) graphs to define new notions which state the relation amid vertices. In this way, the set of vertices are distinguished by another set of vertices.

Definition 2.1. Let $G=(V, \sigma, \mu)$ be a fuzzy(neutrosophic) graph. A vertex $m$ fuzzy(neutrosophic)-resolves vertices $f_{1}$ and $f_{2}$ if $d\left(m, f_{1}\right) \neq d\left(m, f_{2}\right)$. A set $M$ is 
fuzzy(neutrosophic)-resolving set if for every couple of vertices $f_{1}, f_{2} \in V \backslash M$, there's a vertex $m \in M$ such that $m$ fuzzy(neutrosophic)-resolves $f_{1}$ and $f_{2} \cdot|M|$ is called fuzzy(neutrosophic)-metric number of $G$ and

$$
\underset{S \text { is fuzzy(neutrosophic)-resolving set }}{\min } \Sigma_{s \in S} \sigma(s)=\Sigma_{m \in M} \sigma(m)
$$

is called fuzzy(neutrosophic)-metric dimension of $G$ and if

$$
\underset{S \text { is fuzzy(neutrosophic)-resolving set }}{\min } \Sigma_{s \in S} \sigma(s)=\Sigma_{m \in M} \sigma(m)
$$

where $M$ is fuzzy(neutrosophic)-resolving set, then $M$ is called fuzzy(neutrosophic)-metric set of $G$.

Example 2.2. Let $G$ be a fuzzy(neutrosophic) graph as figure (1). By applying Table (3), the 1-set is explored which its cardinality is minimum. $\left\{f_{6}\right\}$ and $\left\{f_{4}\right\}$ are 1 -set which has minimum cardinality amid all sets of vertices but $\left\{f_{4}\right\}$ isn't fuzzy(neutrosophic)-resolving set and $\left\{f_{6}\right\}$ is fuzzy(neutrosophic)-resolving set. Thus there's no fuzzy(neutrosophic)-metric set but $\left\{f_{6}\right\}$. $f_{6}$ fuzzy(neutrosophic)-resolves all given couple of vertices. Therefore one is fuzzy(neutrosophic)-metric number of $G$ and 0.13 is fuzzy(neutrosophic)-metric dimension of $G$. By using Table (3), $f_{4}$ doesn't fuzzy(neutrosophic)-resolve $f_{2}$ and $f_{6}$. $f_{4}$ doesn't fuzzy(neutrosophic)-resolve $f_{1}$ and $f_{5}$, too.

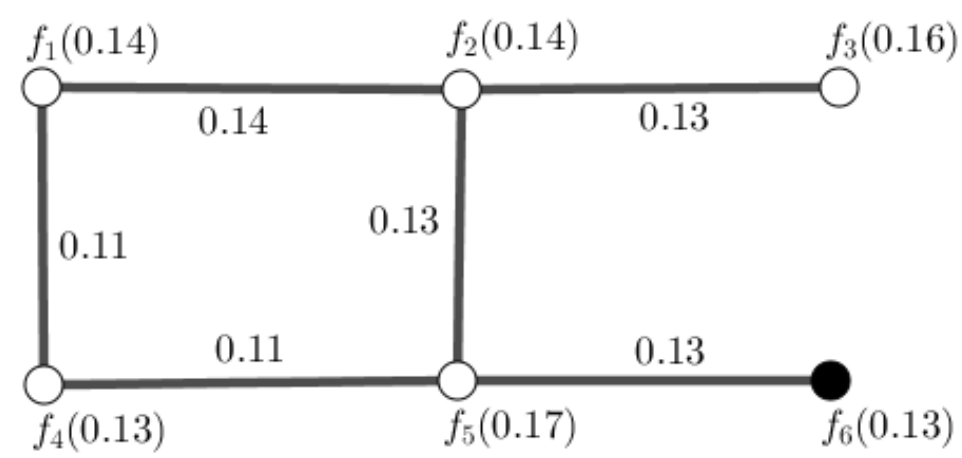

Figure 1. Black vertex $\left\{f_{6}\right\}$ is only fuzzy(neutrosophic)-metric set amid all sets of vertices for fuzzy(neutrosophic) graph $G$.

Table 3. Distances of Vertices from sets of vertices $\left\{f_{6}\right\}$ and $\left\{f_{4}\right\}$ in fuzzy(neutrosophic) Graph $G$.

\begin{tabular}{c|lccccc}
\hline Vertices & $f_{1}$ & $f_{2}$ & $f_{3}$ & $f_{4}$ & $f_{5}$ & $f_{6}$ \\
\hline$f_{6}$ & 0.22 & 0.26 & 0.39 & 0.24 & 0.13 & 0 \\
Vertices & $f_{1}$ & $f_{2}$ & $f_{3}$ & $f_{4}$ & $f_{5}$ & $f_{6}$ \\
$f_{4}$ & 0.11 & 0.24 & 0.37 & 0 & 0.11 & 0.24 \\
\hline
\end{tabular}

Definition 2.3. Consider $\mathcal{G}$ as a family of fuzzy(neutrosophic) graphs on a common vertex set $V$. A vertex $m$ simultaneously fuzzy(neutrosophic)-resolves vertices $f_{1}$ and $f_{2}$ if $d_{G}\left(m, f_{1}\right) \neq d_{G}\left(m, f_{2}\right)$, for all $G \in \mathcal{G}$. A set $M$ is simultaneously

fuzzy(neutrosophic)-resolving set if for every couple of vertices $f_{1}, f_{2} \in V \backslash M$, there's a vertex $m \in M$ such that $m$ resolves $f_{1}$ and $f_{2}$, for all $G \in \mathcal{G} .|M|$ is called simultaneously fuzzy(neutrosophic)-metric number of $\mathcal{G}$ and

$$
\underset{S \text { is fuzzy(neutrosophic)-resolving set }}{\min } \Sigma_{s \in S} \sigma(s)=\Sigma_{m \in M} \sigma(m)
$$


is called simultaneously fuzzy(neutrosophic)-metric dimension of $\mathcal{G}$ and if

$$
\min _{S \text { is fuzzy(neutrosophic)-resolving set }} \Sigma_{s \in S} \sigma(s)=\Sigma_{m \in M} \sigma(m)
$$

where $M$ is fuzzy(neutrosophic)-resolving set, then $M$ is called simultaneously fuzzy(neutrosophic)-metric set of $\mathcal{G}$.

Example 2.4. Let $\mathcal{G}=\left\{G_{1}, G_{2}, G_{3}\right\}$ be a collection of fuzzy(neutrosophic) graphs with common fuzzy(neutrosophic) vertex set and a subset of fuzzy(neutrosophic) edge set as figure (2). By applying Table (4), the 1-set is explored which its cardinality is minimum. $\left\{f_{2}\right\}$ and $\left\{f_{4}\right\}$ are 1-set which has minimum cardinality amid all sets of vertices. $\left\{f_{4}\right\}$ is as fuzzy(neutrosophic)-resolving set as $\left\{f_{6}\right\}$ is. Thus there's no fuzzy(neutrosophic)-metric set but $\left\{f_{4}\right\}$ and $\left\{f_{6}\right\} . f_{6}$ as fuzzy(neutrosophic)-resolves all given couple of vertices as $f_{4}$. Therefore one is fuzzy(neutrosophic)-metric number of $\mathcal{G}$ and 0.13 is fuzzy(neutrosophic)-metric dimension of $\mathcal{G}$. By using Table (4), $f_{4}$ fuzzy(neutrosophic)-resolves all given couple of vertices.

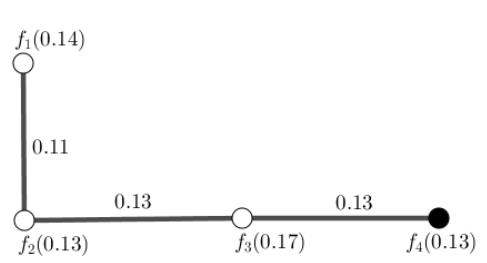

$G_{1}$

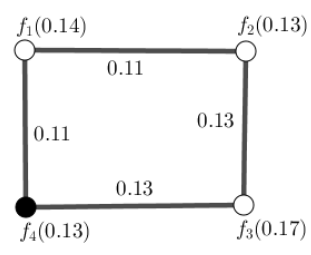

$G_{2}$

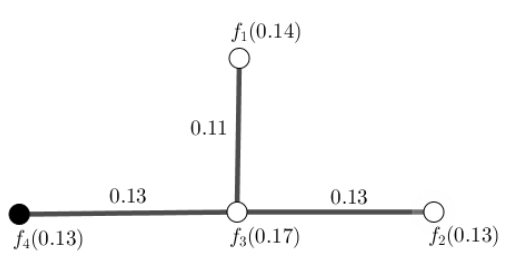

$G_{3}$

Figure 2. Black vertex $\left\{f_{4}\right\}$ and the set of vertices $\left\{f_{2}\right\}$ are simultaneously fuzzy(neutrosophic)-metric set amid all sets of vertices for family of fuzzy(neutrosophic) graphs $\mathcal{G}$.

Table 4. Distances of Vertices from set of vertices $\left\{f_{6}\right\}$ in Family of fuzzy(neutrosophic) Graphs $\mathcal{G}$.

\begin{tabular}{c|lccc}
\hline Vertices of $G_{1}$ & $f_{1}$ & $f_{2}$ & $f_{3}$ & $f_{4}$ \\
\hline$f_{4}$ & 0.37 & 0.26 & 0.13 & 0 \\
Vertices of $G_{2}$ & $f_{1}$ & $f_{2}$ & $f_{3}$ & $f_{4}$ \\
$f_{4}$ & 0.11 & 0.22 & 0.13 & 0 \\
Vertices of $G_{3}$ & $f_{1}$ & $f_{2}$ & $f_{3}$ & $f_{4}$ \\
$f_{4}$ & 0.24 & 0.26 & 0.13 & 0 \\
\hline
\end{tabular}

\section{General Relationships}

Proposition 3.1. Let $G$ be a fuzzy(neutrosophic) path. Then every leaf is fuzzy(neutrosophic)-resolving set.

Proof. Let $l$ be a leaf. For every given a couple of vertices $f_{i}$ and $f_{j}$, we get $d\left(l, f_{i}\right) \neq d\left(l, f_{j}\right)$. Since if we reassign indexes to vertices such that every vertex $f_{i}$ and $l$ have $i$ vertices amid themselves, then $d\left(l, f_{i}\right)=\Sigma_{j \leq i} \mu\left(f_{j} f_{i}\right) \leq i$. Thus $j \leq i$ implies

$\Sigma_{t \leq j} \mu\left(f_{t} f_{j}\right)+\Sigma_{j \leq s \leq i} \mu\left(f_{s} f_{i}\right)>\Sigma_{j \leq i} \mu\left(f f_{i}\right) \equiv d\left(l, f_{j}\right)+c=d\left(l, f_{i}\right) \equiv d\left(l, f_{j}\right)<d\left(l, f_{i}\right)$.

Therefore, by $d\left(l, f_{j}\right)<d\left(l, f_{i}\right)$, we get $d\left(l, f_{i}\right) \neq d\left(l, f_{j}\right) . f_{i}$ and $f_{j}$ are arbitrary so $l$ fuzzy(neutrosophic)-resolves any given couple of vertices $f_{i}$ and $f_{j}$ which implies $\{l\}$ is a fuzzy(neutrosophic)-resolving set. 
Corollary 3.2. Let $G$ be a fixed-edge fuzzy(neutrosophic) path. Then every leaf is fuzzy(neutrosophic)-resolving set.

Proof. Let $l$ be a leaf. For every given couple of vertices, $f_{i}$ and $f_{j}$, we get $d\left(l, f_{i}\right)=c i \neq d\left(l, f_{j}\right)=c j$. It implies $l$ fuzzy(neutrosophic)-resolves any given couple of vertices $f_{i}$ and $f_{j}$ which implies $\{l\}$ is a fuzzy(neutrosophic)-resolving set.

Corollary 3.3. Let $G$ be a fixed-vertex fuzzy(neutrosophic) path. Then every leaf is fuzzy(neutrosophic)-metric set, fuzzy(neutrosophic)-metric number is one and fuzzy(neutrosophic)-metric dimension is c where $c=\sigma(f), f \in V$.

Proof. By Proposition (3.1), every leaf is fuzzy(neutrosophic)-resolving set. By $c=\sigma(f), \forall f \in V$, every leaf is fuzzy(neutrosophic)-metric set,

fuzzy(neutrosophic)-metric number is one and fuzzy(neutrosophic)-metric dimension is $c$.

Proposition 3.4. Let $G$ be a fuzzy(neutrosophic) path. Then a set including every couple of vertices is fuzzy(neutrosophic)-resolving set.

Proof. Let $f$ and $f^{\prime}$ be a couple of vertices. For every given a couple of vertices $f_{i}$ and $f_{j}$, we get either $d\left(f, f_{i}\right) \neq d\left(f, f_{j}\right)$ or $d\left(f^{\prime}, f_{i}\right) \neq d\left(f^{\prime}, f_{j}\right)$.

Corollary 3.5. Let $G$ be a fixed-edge fuzzy(neutrosophic) path. Then every set containing couple of vertices is fuzzy(neutrosophic)-resolving set.

Proof. Consider $G$ is a fuzzy(neutrosophic) path. Thus by Proposition (3.4), every set containing couple of vertices is fuzzy(neutrosophic)-resolving set. So it holds for any given fixed-edge path fuzzy(neutrosophic) graph.

\section{Fuzzy(Neutrosophic) Twin Vertices}

Proposition 4.1. Let $G$ be a fuzzy(neutrosophic) graph. An $(k-1)$-set from an $k$-set of fuzzy(neutrosophic) twin vertices is subset of a fuzzy(neutrosophic)-resolving set.

Proof. If $t$ and $t^{\prime}$ are fuzzy(neutrosophic) twin vertices, then $N(t)=N\left(t^{\prime}\right)$ and $\mu(t s)=\mu\left(t^{\prime} s\right)$, for all $s \in N(t)=N\left(t^{\prime}\right)$.

Corollary 4.2. Let $G$ be a fuzzy(neutrosophic) graph. The number of fuzzy(neutrosophic) twin vertices is $n-1$. Then fuzzy(neutrosophic)-metric number is $n-2$.

Proof. Let $f$ and $f^{\prime}$ be two vertices. By supposition, the cardinality of set of fuzzy(neutrosophic) twin vertices is $n-2$. Thus there are two cases. If both are fuzzy(neutrosophic) twin vertices, then $N(f)=N\left(f^{\prime}\right)$ and $\mu(f s)=\mu\left(f^{\prime} s^{\prime}\right), \forall s \in N(f)$, $\forall s^{\prime} \in N\left(f^{\prime}\right)$. It implies $d(f, t)=d(f, t)$ for all $t \in V$. Thus suppose if not, then let $f$ be a vertex which isn't fuzzy(neutrosophic) twin vertices with any given vertex and let $f^{\prime}$ be a vertex which is fuzzy(neutrosophic) twin vertices with any given vertex but not $f$. By supposition, it's possible and this is only case. Therefore, any given distinct vertex fuzzy(neutrosophic)-resolves $f$ and $f^{\prime}$. Then $V \backslash\left\{f, f^{\prime}\right\}$ is fuzzy(neutrosophic)-resolving set. It implies fuzzy(neutrosophic)-metric number is $n-2$.

Corollary 4.3. Let $G$ be a fuzzy(neutrosophic) graph. The number of fuzzy(neutrosophic) twin vertices is $n$. Then $G$ is fixed-edge fuzzy(neutrosophic) graph. 
Proof. Suppose $f$ and $f^{\prime}$ are two given edges. By supposition, every couple of vertices are fuzzy(neutrosophic) twin vertices. It implies $\mu(f)=\mu\left(f^{\prime}\right) . f$ and $f^{\prime}$ are arbitrary so every couple of edges have same values. It induces $G$ is fixed-edge fuzzy(neutrosophic) graph.

Corollary 4.4. Let $G$ be a fixed-vertex fuzzy(neutrosophic) graph. The number of fuzzy(neutrosophic) twin vertices is $n-1$. Then fuzzy(neutrosophic)-metric number is $n-2$, fuzzy(neutrosophic)-metric dimension is $(n-2) \sigma(m)$ where $m$ is fuzzy(neutrosophic) twin vertex with a vertex. Every $(n-2)$-set including fuzzy(neutrosophic) twin vertices is fuzzy(neutrosophic)-metric set.

Proof. By Corollary (4.2), fuzzy(neutrosophic)-metric number is $n-2$. By $G$ is a fixed-vertex fuzzy(neutrosophic) graph, fuzzy metric dimension is $(n-2) \sigma(m)$ where $m$ is fuzzy(neutrosophic) twin vertex with a vertex. One vertex doesn't belong to set of fuzzy(neutrosophic) twin vertices and a vertex from that set, are out of fuzzy metric set. It induces every $(n-2)$-set including fuzzy(neutrosophic) twin vertices is fuzzy metric set.

Proposition 4.5. Let $G$ be a fixed-vertex fuzzy(neutrosophic) graph such that it's fuzzy(neutrosophic) complete. Then fuzzy(neutrosophic)-metric number is $n-1$, fuzzy(neutrosophic)-metric dimension is $(n-1) \sigma(m)$ where $m$ is a given vertex. Every $(n-1)$-set is fuzzy(neutrosophic)-metric set.

Proof. In fuzzy(neutrosophic) complete, every couple of vertices are twin vertices. By $G$ is a fixed-vertex fuzzy(neutrosophic) graph and it's fuzzy(neutrosophic) complete, every couple of vertices are fuzzy(neutrosophic) twin vertices. Thus by Proposition (4.1), the result follows.

Proposition 4.6. Let $\mathcal{G}$ be a family of fuzzy(neutrosophic) graphs with common vertex set. Then simultaneously fuzzy(neutrosophic)-metric number of $\mathcal{G}$ is $n-1$.

Proof. Consider $(n-1)$-set. Thus there's no couple of vertices to be fuzzy(neutrosophic)-resolved. Therefore, every $(n-1)$-set is fuzzy(neutrosophic)-resolving set for any given fuzzy(neutrosophic) graph. Then it holds for any fuzzy(neutrosophic) graph. It implies it's fuzzy(neutrosophic)-resolving set and its cardinality is fuzzy(neutrosophic)-metric number. $(n-1)$-set has the cardinality $n-1$. Then it holds for any fuzzy(neutrosophic) graph. It induces it's simultaneously fuzzy(neutrosophic)-resolving set and its cardinality is simultaneously fuzzy(neutrosophic)-metric number.

Proposition 4.7. Let $\mathcal{G}$ be a family of fuzzy(neutrosophic) graphs with common vertex set. Then simultaneously fuzzy(neutrosophic)-metric number of $\mathcal{G}$ is greater than the maximum fuzzy(neutrosophic)-metric number of $G \in \mathcal{G}$.

Proof. Suppose $t$ and $t^{\prime}$ are simultaneously fuzzy(neutrosophic)-metric number of $\mathcal{G}$ and fuzzy(neutrosophic)-metric number of $G \in \mathcal{G}$. Thus $t$ is fuzzy(neutrosophic)-metric number for any $G \in \mathcal{G}$. Hence, $t \geq t^{\prime}$. So simultaneously fuzzy(neutrosophic)-metric number of $\mathcal{G}$ is greater than the maximum fuzzy(neutrosophic)-metric number of $G \in \mathcal{G}$.

Proposition 4.8. Let $\mathcal{G}$ be a family of fuzzy(neutrosophic) graphs with common vertex set. Then simultaneously fuzzy(neutrosophic)-metric number of $\mathcal{G}$ is greater than simultaneously fuzzy(neutrosophic)-metric number of $\mathcal{H} \subseteq \mathcal{G}$. 
Proof. Suppose $t$ and $t^{\prime}$ are simultaneously fuzzy(neutrosophic)-metric number of $\mathcal{G}$ and $\mathcal{H}$. Thus $t$ is fuzzy(neutrosophic)-metric number for any $G \in \mathcal{G}$. It implies $t$ is fuzzy(neutrosophic)-metric number for any $G \in \mathcal{H}$. So $t$ is simultaneously fuzzy(neutrosophic)-metric number of $\mathcal{H}$. By applying Definition about being the minimum number, $t \geq t^{\prime}$. So simultaneously fuzzy(neutrosophic)-metric number of $\mathcal{G}$ is greater than simultaneously fuzzy(neutrosophic)-metric number of $\mathcal{H} \subseteq \mathcal{G}$.

Theorem 4.9. Fuzzy(neutrosophic) twin vertices aren't fuzzy(neutrosophic)-resolved in any given fuzzy(neutrosophic) graph.

Proof. Let $t$ and $t^{\prime}$ be fuzzy(neutrosophic) twin vertices. Then $N(t)=N\left(t^{\prime}\right)$ and $\mu(t s)=\mu\left(t^{\prime} s\right)$, for all $s, s^{\prime} \in V$ such that $t s, t^{\prime} s \in E$. Thus for every given vertex $s^{\prime} \in V, d_{G}\left(s^{\prime}, t\right)=d_{G}(s, t)$ where $G$ is a given fuzzy(neutrosophic) graph. It means that $t$ and $t^{\prime}$ aren't resolved in any given fuzzy(neutrosophic) graph. $t$ and $t^{\prime}$ are arbitrary so fuzzy(neutrosophic) twin vertices aren't resolved in any given fuzzy(neutrosophic) graph.

Proposition 4.10. Let $G$ be a fixed-vertex fuzzy(neutrosophic) graph. If $G$ is fuzzy(neutrosophic) complete, then every couple of vertices are fuzzy(neutrosophic) twin vertices.

Proof. Let $t$ and $t^{\prime}$ be couple of given vertices. By $G$ is fuzzy(neutrosophic) complete, $N(t)=N\left(t^{\prime}\right)$. By $G$ is a fixed-vertex fuzzy(neutrosophic) graph, $\mu(t s)=\mu\left(t^{\prime} s\right)$, for all edges $t s, t^{\prime} s \in E$. Thus $t$ and $t^{\prime}$ are fuzzy(neutrosophic) twin vertices. $t$ and $t^{\prime}$ are arbitrary couple of vertices, hence every couple of vertices are fuzzy(neutrosophic) twin vertices.

Theorem 4.11. Let $\mathcal{G}$ be a family of fuzzy(neutrosophic) graphs with common vertex set and $G \in \mathcal{G}$ is a fixed-vertex fuzzy(neutrosophic) graph such that it's

fuzzy(neutrosophic) complete. Then simultaneously fuzzy(neutrosophic)-metric number is $n-1$, simultaneously fuzzy(neutrosophic)-metric dimension is $(n-1) \sigma(m)$ where $m$ is a given vertex. Every $(n-1)$-set is simultaneously fuzzy(neutrosophic)-metric set for $\mathcal{G}$.

Proof. $G$ is fixed-vertex fuzzy(neutrosophic) graph and it's fuzzy(neutrosophic) complete. So by Theorem (4.10), we get every couple of vertices in fuzzy(neutrosophic) complete are fuzzy(neutrosophic) twin vertices. So every couple of vertices, by Theorem (4.9), aren't resolved.

Corollary 4.12. Let $\mathcal{G}$ be a family of fuzzy(neutrosophic) graphs with fuzzy(neutrosophic) common vertex set and $G \in \mathcal{G}$ is a fuzzy(neutrosophic) complete. Then simultaneously fuzzy(neutrosophic)-metric number is $n-1$, simultaneously fuzzy(neutrosophic)-metric dimension is $(n-1) \sigma(m)$ where $m$ is a given vertex. Every $(n-1)$-set is simultaneously fuzzy(neutrosophic)-metric set for $\mathcal{G}$.

Proof. By fuzzy(neutrosophic) graphs with fuzzy(neutrosophic) common vertex set, $G$ is fixed-vertex fuzzy(neutrosophic) graph. It's fuzzy(neutrosophic) complete. So by Theorem (4.11), we get intended result.

Theorem 4.13. Let $\mathcal{G}$ be a family of fuzzy(neutrosophic) graphs with common vertex set and for every given couple of vertices, there's a $G \in \mathcal{G}$ such that in that, they're fuzzy(neutrosophic) twin vertices. Then simultaneously fuzzy(neutrosophic)-metric number is $n-1$, simultaneously fuzzy(neutrosophic)-metric dimension is $(n-1) \sigma(m)$ where $m$ is a given vertex. Every $(n-1)$-set is simultaneously fuzzy(neutrosophic)-metric set for $\mathcal{G}$. 
Proof. By Proposition (4.6), simultaneously fuzzy(neutrosophic)-metric number is $n-1$. By Theorem (4.9), simultaneously fuzzy(neutrosophic)-metric dimension is $(n-1) \sigma(m)$ where $m$ is a given vertex. Also, every $(n-1)$-set is simultaneously fuzzy(neutrosophic)-metric set for $\mathcal{G}$.

Theorem 4.14. Let $\mathcal{G}$ be a family of fuzzy(neutrosophic) graphs with common vertex set. If $\mathcal{G}$ contains three fixed-vertex fuzzy(neutrosophic) stars with different center, then simultaneously fuzzy(neutrosophic)-metric number is $n-2$, simultaneously fuzzy(neutrosophic)-metric dimension is $(n-2) \sigma(m)$ where $m$ is a given vertex. Every $(n-2)$-set is simultaneously fuzzy(neutrosophic)-metric set for $\mathcal{G}$.

Proof. The cardinality of set of fuzzy(neutrosophic) twin vertices is $n-1$. Thus by Corollary (4.4), the result follows.

Corollary 4.15. Let $\mathcal{G}$ be a family of fuzzy(neutrosophic) graphs with fuzzy(neutrosophic) common vertex set. If $\mathcal{G}$ contains three fuzzy(neutrosophic) stars with different center, then simultaneously fuzzy(neutrosophic)-metric number is $n-2$, simultaneously fuzzy(neutrosophic)-metric dimension is $(n-2) \sigma(m)$ where $m$ is a given vertex. Every $(n-2)$-set is simultaneously fuzzy(neutrosophic)-metric set for $\mathcal{G}$.

Proof. By fuzzy(neutrosophic) graphs with fuzzy(neutrosophic) common vertex set, $G$ is fixed-vertex fuzzy(neutrosophic) graph. It's fuzzy(neutrosophic) complete. So by Theorem (4.14), we get intended result.

\section{Antipodal Vertices}

\subsection{Even Fuzzy(Neutrosophic) Cycle}

Proposition 5.1. Consider two antipodal vertices $x$ and $y$ in any given fixed-edge even fuzzy(neutrosophic) cycle. Let $u$ and $v$ be given vertices. Then $d(x, u) \neq d(x, v)$ if and only if $d(y, u) \neq d(y, v)$.

Proof. $(\Rightarrow)$. Consider $d(x, u) \neq d(x, v)$. By

$d(x, u)+d(u, y)=d(x, y)=D(G), D(G)-d(x, u) \neq D(G)-d(x, v)$. It implies $d(y, u) \neq d(y, v)$.

$(\Leftarrow)$. Consider $d(y, u) \neq d(y, v)$. By

$d(y, u)+d(u, x)=d(x, y)=D(G), D(G)-d(y, u) \neq D(G)-d(y, v)$. It implies $d(x, u) \neq d(x, v)$.

Proposition 5.2. Consider two antipodal vertices $x$ and $y$ in any given fixed-edge even fuzzy(neutrosophic) cycle. Let $u$ and $v$ be given vertices. Then $d(x, u)=d(x, v)$ if and only if $d(y, u)=d(y, v)$.

Proof. $(\Rightarrow)$. Consider $d(x, u)=d(x, v)$. By

$d(x, u)+d(u, y)=d(x, y)=D(G), D(G)-d(x, u)=D(G)-d(x, v)$. It implies $d(y, u)=d(y, v)$.

$(\Leftarrow)$. Consider $d(y, u)=d(y, v)$. By

$d(y, u)+d(u, x)=d(x, y)=D(G), D(G)-d(y, u)=D(G)-d(y, v)$. It implies $d(x, u)=d(x, v)$.

Proposition 5.3. The set contains two antipodal vertices, isn't fuzzy(neutrosophic)-metric set in any given fixed-edge even fuzzy(neutrosophic) cycle. 
Proof. Let $x$ and $y$ be two given antipodal vertices in any given even fuzzy(neutrosophic) cycle. By Proposition (5.1), $d(x, u) \neq d(x, v)$ if and only if $d(y, u) \neq d(y, v)$. It implies that if $x$ fuzzy(neutrosophic)-resolves a couple of vertices, then $y$ fuzzy(neutrosophic)-resolves them, too. Thus either $x$ is in fuzzy(neutrosophic)-metric set or $y$ is. It induces the set contains two antipodal vertices, isn't fuzzy(neutrosophic)-metric set in any given even fuzzy(neutrosophic) cycle.

Proposition 5.4. Consider two antipodal vertices $x$ and $y$ in any given fixed-edge even fuzzy(neutrosophic) cycle. $x$ fuzzy(neutrosophic)-resolves a given couple of vertices, $z$ and $z^{\prime}$, if and only if $y$ does.

Proof. $(\Rightarrow) . x$ fuzzy(neutrosophic)-resolves a given couple of vertices, $z$ and $z^{\prime}$, then $d(x, z) \neq d\left(x, z^{\prime}\right)$. By Proposition (5.1), $d(x, z) \neq d\left(x, z^{\prime}\right)$ if and only if $d(y, z) \neq d\left(y, z^{\prime}\right)$. Thus $y$ fuzzy(neutrosophic)-resolves a given couple of vertices $z$ and $z^{\prime}$.

$(\Leftarrow)$. $y$ fuzzy(neutrosophic)-resolves a given couple of vertices, $z$ and $z^{\prime}$, then $d(y, z) \neq d\left(y, z^{\prime}\right)$. By Proposition (5.1), $d(y, z) \neq d\left(y, z^{\prime}\right)$ if and only if $d(x, z) \neq d\left(x, z^{\prime}\right)$. Thus $x$ fuzzy(neutrosophic)-resolves a given couple of vertices $z$ and $z^{\prime}$.

Proposition 5.5. There are two antipodal vertices aren't fuzzy(neutrosophic)-resolved by other two antipodal vertices in any given fixed-edge even fuzzy(neutrosophic) cycle.

Proof. Suppose $x$ and $y$ are a couple of vertices. It implies $d(x, y)=D(G)$. Consider $u$ and $v$ are another couple of vertices such that $d(x, u)=\frac{D(G)}{2}$. It implies $d(y, u)=\frac{D(G)}{2}$. Thus $d(x, u)=d(y, u)$. Therefore, $u$ doesn't fuzzy(neutrosophic)-resolve a given couple of vertices $x$ and $y$. By $D(G)=d(u, v)=d(u, x)+d(x, v)=\frac{D(G)}{2}+d(x, v)$, $d(x, v)=\frac{D(G)}{2}$. It implies $d(y, v)=\frac{D(G)}{2}$. Thus $d(x, v)=d(y, v)$. Therefore, $v$ doesn't fuzzy(neutrosophic)-resolve a given couple of vertices $x$ and $y$.

Proposition 5.6. For any two antipodal vertices in any given fixed-edge even fuzzy(neutrosophic) cycle, there are only two antipodal vertices don't fuzzy(neutrosophic)-resolve them

Proof. Suppose $x$ and $y$ are a couple of vertices such that they're antipodal vertices. Let $u$ be a vertex such that $d(x, u)=\frac{D(G)}{2}$. It implies $d(y, u)=\frac{D(G)}{2}$. Thus $d(x, u)=d(y, u)$. Therefore, $u$ doesn't fuzzy(neutrosophic)-resolve a given couple of vertices $x$ and $y$. Let $v$ be a antipodal vertex for $u$ such that $u$ and $v$ are antipodal vertices. Thus $v d(x, v)=\frac{D(G)}{2}$. It implies $d(y, v)=\frac{D(G)}{2}$. Therefore, $v$ doesn't fuzzy(neutrosophic)-resolve a given couple of vertices $x$ and $y$. If $u$ is a vertex such that $d(x, u) \neq \frac{D(G)}{2}$ and $v$ is a vertex such that $u$ and $v$ are antipodal vertices. Thus $d(x, v) \neq \frac{D(G)}{2}$ It induces either $d(x, u) \neq d(y, u)$ or $d(x, v) \neq d(y, v)$. It means either $u$ fuzzy(neutrosophic)-resolves a given couple of vertices $x$ and $y$ or $v$ fuzzy(neutrosophic)-resolves a given couple of vertices $x$ and $y$.

Proposition 5.7. In any given fixed-edge even fuzzy(neutrosophic) cycle, for any vertex, there's only one vertex such that they're antipodal vertices.

Proof. If $d(x, y)=D(G)$, then $x$ and $y$ are antipodal vertices.

Proposition 5.8. Let $G$ be a fixed-edge even fuzzy(neutrosophic) cycle. Then every couple of vertices are fuzzy(neutrosophic)-resolving set if and only if they aren't antipodal vertices.

Proof. If $x$ and $y$ are antipodal vertices, then they don't fuzzy(neutrosophic)-resolve a given couple of vertices $u$ and $v$ such that they're antipodal vertices and $d(x, u)=\frac{D(G)}{2}$. Since $d(x, u)=d(x, v)=d(y, u)=d(y, v)=\frac{D(G)}{2}$. 
Corollary 5.9. Let $G$ be a fixed-edge even fuzzy(neutrosophic) cycle. Then fuzzy(neutrosophic)-metric number is two.

Proof. A set contains one vertex $x$ isn't fuzzy(neutrosophic)-resolving set. Since it doesn't fuzzy(neutrosophic)-resolve a given couple of vertices $u$ and $v$ such that $d(x, u)=d(x, v)=1$. Thus fuzzy(neutrosophic)-metric number $\geq 2$. By Proposition (5.8), every couple of vertices such that they aren't antipodal vertices, are fuzzy(neutrosophic)-resolving set. Therefore, fuzzy(neutrosophic)-metric number is 2.

Corollary 5.10. Let $G$ be a fixed-edge even fuzzy(neutrosophic) cycle. Then fuzzy(neutrosophic)-metric set contains couple of vertices such that they aren't antipodal vertices.

Proof. By Corollary (5.9), fuzzy(neutrosophic)-metric number is two. By Proposition (5.8), every couple of vertices such that they aren't antipodal vertices, are fuzzy(neutrosophic)-resolving set. Therefore, fuzzy(neutrosophic)-metric set contains couple of vertices such that they aren't antipodal vertices.

Corollary 5.11. Let $\mathcal{G}$ be a family of fixed-edge odd fuzzy(neutrosophic) cycles with fuzzy(neutrosophic) common vertex set. Then simultaneously fuzzy(neutrosophic)-metric set contains couple of vertices such that they aren't antipodal vertices and fuzzy(neutrosophic)-metric number is two.

\subsection{Odd Fuzzy(Neutrosophic) Cycle}

Proposition 5.12. In any given fixed-edge odd fuzzy(neutrosophic) cycle, for any vertex, there's no vertex such that they're antipodal vertices.

Proof. Let $G$ be a fixed-edge odd fuzzy(neutrosophic) cycle. if $x$ is a given vertex. Then there are two vertices $u$ and $v$ such that $d(x, u)=d(x, v)=D(G)$. It implies they aren't antipodal vertices.

Proposition 5.13. Let $G$ be a fixed-edge odd fuzzy(neutrosophic) cycle. Then every couple of vertices are fuzzy(neutrosophic)-resolving set.

Proof. Let $l$ and $l^{\prime}$ be couple of vertices. Thus, by Proposition (5.12), $l$ and $l^{\prime}$ aren't antipodal vertices. It implies for every given couple of vertices $f_{i}$ and $f_{j}$, we get either $d\left(l, f_{i}\right) \neq d\left(l, f_{j}\right)$ or $d\left(l^{\prime}, f_{i}\right) \neq d\left(l^{\prime}, f_{j}\right)$. Therefore, $f_{i}$ and $f_{j}$ are fuzzy(neutrosophic)-resolved by either $l$ or $l^{\prime}$. It induces the set $\left\{l, l^{\prime}\right\}$ is fuzzy(neutrosophic)-resolving set.

Proposition 5.14. Let $G$ be a fixed-edge odd fuzzy(neutrosophic) cycle. Then fuzzy(neutrosophic)-metric number is two.

Proof. Let $l$ and $l^{\prime}$ be couple of vertices. Thus, by Proposition (5.12), $l$ and $l^{\prime}$ aren't antipodal vertices. It implies for every given couple of vertices $f_{i}$ and $f_{j}$, we get either $d\left(l, f_{i}\right) \neq d\left(l, f_{j}\right)$ or $d\left(l^{\prime}, f_{i}\right) \neq d\left(l^{\prime}, f_{j}\right)$. Therefore, $f_{i}$ and $f_{j}$ are fuzzy(neutrosophic)-resolved by either $l$ or $l^{\prime}$. It induces the set $\left\{l, l^{\prime}\right\}$ is fuzzy(neutrosophic)-resolving set.

Corollary 5.15. Let $G$ be a fixed-edge odd fuzzy(neutrosophic) cycle. Then fuzzy(neutrosophic)-metric set contains couple of vertices.

Proof. By Proposition (5.14), fuzzy(neutrosophic)-metric number is two. By Proposition (5.13), every couple of vertices are fuzzy(neutrosophic)-resolving set. Therefore, fuzzy(neutrosophic)-metric set contains couple of vertices. 
Corollary 5.16. Let $\mathcal{G}$ be a family of fixed-edge odd fuzzy(neutrosophic) cycles with fuzzy(neutrosophic) common vertex set. Then simultaneously fuzzy(neutrosophic)-metric set contains couple of vertices and fuzzy(neutrosophic)-metric number is two.

\section{Extended Results}

Proposition 6.1. If we use fixed-vertex strong fuzzy(neutrosophic) cycles instead of fixed-edge fuzzy(neutrosophic) cycles, then all results of Section (5) hold.

Proof. Let $G$ be a fixed-vertex strong fuzzy(neutrosophic) cycles. By $G$ is fuzzy(neutrosophic) strong and it's fixed-vertex, $G$ is fixed-edge fuzzy(neutrosophic).

\section{References}

1. M. Akram, and G. Shahzadi, Operations on Single-Valued Neutrosophic Graphs, Journal of uncertain systems 11 (1) (2017) 1-26.

2. K. Atanassov, Intuitionistic fuzzy sets, Fuzzy Sets Syst. 20 (1986) 87-96.

3. S. Broumi, M. Talea, A. Bakali and F. Smarandache, Single-valued neutrosophic graphs, Journal of New Theory 10 (2016) 86-101.

4. N. Shah, and A. Hussain, Neutrosophic soft graphs, Neutrosophic Set and Systems 11 (2016) 31-44.

5. Henry Garrett, Big Sets Of Vertices, Preprints 2021, 2021060189 (doi: 10.20944/preprints202106.0189.v1).

6. Henry Garrett, Locating And Location Number, Preprints 2021, 2021060206 (doi: 10.20944/preprints202106.0206.v1).

7. Henry Garrett, Metric Dimensions Of Graphs, Preprints 2021, 2021060392 (doi: 10.20944/preprints202106.0392.v1).

8. Henry Garrett, New Graph Of Graph, Preprints 2021, 2021060323 (doi: 10.20944/preprints202106.0323.v1).

9. Henry Garrett, Numbers Based On Edges, Preprints 2021, 2021060315 (doi: 10.20944/preprints202106.0315.v1).

10. Henry Garrett, Matroid And Its Outlines, Preprints 2021, 2021060146 (doi: 10.20944/preprints202106.0146.v1).

11. Henry Garrett, Matroid And Its Relations, Preprints 2021, 2021060080 (doi: 10.20944/preprints202106.0080.v1).

12. A. Shannon and K.T. Atanassov, A first step to a theory of the intuitionistic fuzzy graphs, Proceeding of FUBEST (Lakov, D., Ed.) Sofia (1994) 59-61.

13. F. Smarandache, A Unifying field in logics neutrosophy: Neutrosophic probability, set and logic, Rehoboth: American Research Press (1998).

14. H. Wang, F. Smarandache, Y. Zhang, and R. Sunderraman, Single-valued neutrosophic sets, Multispace and Multistructure 4 (2010) 410-413.

15. L. A. Zadeh, Fuzzy sets, Information and Control 8 (1965) 338-353. 